\title{
Granulomatosis con Poliangitis: presentación de un caso
}

\author{
Granulomatosis with Polyangiitis: a case report \\ Nataly Sofía Valdiviezo Allauca. ${ }^{1}$, Mayra Silvana Chávez Berrones. ${ }^{2}$ \& Cynthia Desire \\ Merino Guerrero. ${ }^{3}$
}

Recibido: 16-06-2019 / Revisado: 20-07-209 /Aceptado: 13-08-2019/ Publicado: 06-09-2019

\begin{abstract}
.
DOI: https://doi.org/10.33262/cienciadigital.v3i3.3.821

Wegener's Granulomatosis $(\mathrm{GW})$ is a vasculitis of small to medium vessels of unknown etiology, associated with anti- neutrophil cytoplasmic antibodies (ANCA). The main characteristic is a necrotizing vasculitis, with formation of granulomas. Its classic clinical pattern is a triad that involves the upper airways, lungs and kidneys; the definitive diagnosis is reached by histopathological result. In this article, a clinical case of a patient diagnosed with Wegener's Vasculitis is detailed incidentally by performing a confirmatory lung biopsy performed at the Pneumology Service of the Specialties Hospital N 1 FFAA Quito, Ecuador, with the patient's prior informed consent. Presentation of the case: A 67-year-old male patient presented with a clinical picture characterized by a cough without expectoration and a progressive weight loss of $10 \mathrm{~kg}$ over two months. Underwent bronchoscopy with findings of infiltration of the entire right bronchial mucosa with cytology, lavage and bronchial biopsy suspicious of malignancy, however after open lung biopsy is confirmed Wegener's Vasculitis beginning treatment with cyclophosphamide 6 cycles with good evolution clinic. Conclusions: Wegener's Granulomatosis is a multisystem disorder, which can be related to an important morbidity and mortality. Although GW has been associated with the presence of positive ANCA, it is not always indicative of disease, as is the case described in this article. Therefore, it is essential to perform histological examination of the lesioned tissue to confirm the diagnosis.
\end{abstract}

\footnotetext{
${ }^{1}$ Médico residente de Neumología de Hospital de Especialidades de las Fuerzas Armadas No 1, n.atthy89@hotmail.com

${ }^{2}$ Médico residente de Neumología de Hospital de Especialidades de las Fuerzas Armadas No 1, mayra_mschb@live.com

${ }^{3}$ Médico residente de Unidad de Cuidados Intensivos de Hospital de Especialidades de las Fuerzas Armadas No 1, cynthiamerinog@gmail.com
} 
Keywords: Granulomatosis with Polyangiitis, Pulmonary Biopsy, clophosphamide

\section{Resumen}

La granulomatosis con poliangitis (GP) es una vasculitis de pequeños a medianos vasos de etiología desconocida, asociada con anticuerpos anticitoplasma del neutrófilo (ANCA). Como característica principal es una vasculitis necrotizante, con formación de granulomas. Su patrón clínico clásico es una triada que involucra vía aérea superior, pulmones y riñones. El diagnóstico definitivo se realiza mediante histopatología. Se presenta el caso de un paciente adulto mayor quien tiene como único antecedente una Hiperplasia Prostática benigna en tratamiento, quien acude con cuadro clínico de 2 meses de evolución de tos sin expectoración y pérdida de peso de 10 kilogramos, el mismo fue sometido a Broncoscopia con hallazgos de infiltración de toda la mucosa bronquial de predominio derecho, con citología, lavado y biopsia bronquial sospechosos de malignidad, realizándose biopsia pulmonar abierta misma en la que por histopatología confirma el diagnóstico de GP, iniciándose tratamiento con Ciclofosfamida con buena respuesta.

Palabras claves: Granulomatosis con Poliangitis, Biopsia Pulmonar, Ciclofosfamida

\section{Introducción}

La granulomatosis con poliangitis (GP), conocida como previamente como granulomatosis de Wegener, es una vasculitis multisistémica que involucra preferentemente vasos de tamaño pequeño a mediano. Su tríada histológica típica consiste en necrosis tisular, vasculitis e inflamación granulomatosa. Si no se trata, la mortalidad en el plazo de un año desde el diagnóstico es del $90 \%$ la etiología exacta es aún desconocida. ${ }^{(1) .}$

En Latinoamérica se han publicado 750 artículos, 10 de los cuales son estudios de casos de México, Brasil, Chile y Colombia; sin embargo, no existen investigaciones donde se evalúe, en términos de prevalencia e incidencia, las vasculitis primarias en Latinoamérica.

Según lo observado, la distribución epidemiológica registrada demuestra que la vasculitis de Takayasu es la más frecuente en países como Brasil, Colombia y México; mientras que en Chile y Perú son la GP y la Poliangitis microscópica (PAM), respectivamente. No existen evidencias de datos históricos de estos países, excepto los estudios realizados en la última década. ${ }^{(2)}$. En Ecuador se han reportado 3 casos GP siendo descritas 1 en Quito, 1 en Guayaquil y 1 en Ambato ${ }^{(3)}$

La edad al diagnóstico es entre 45 y 60 años. Los hombres y las mujeres se ven afectados con frecuencia similar. En individuos de raza negra, así como en niños pueden ocurrir casos raros de GP.

La afección pulmonar abarca desde nódulos pulmonares asintomáticos a infiltrados pulmonares y hemorragias alveolares fulminantes. El pronóstico es malo debido a insuficiencia renal o respiratoria, aunque las cifras han cambiado gracias al tratamiento con 
glucocorticoides (GC) y CYC. Es una enfermedad con baja incidencia en urgencias, donde la anamnesis debe ser dirigida y apoyada por pruebas complementarias precisas. ${ }^{(4)}$ En general, el tratamiento de este tipo de patología se basa en dosis altas de GC más CYC y desde 2010, Rituximab (RTX) para la remisión por inducción. ${ }^{(5)}$

Se presenta como caso un paciente adulto mayor con tos seca de dos meses de evolución acompañada de síndrome constitucional.

\section{PRESENTACIÓN DE CASO}

Se trata de un paciente masculino de 67 años de edad, residente en Chimborazo, Ecuador, de profesión militar retirado, con antecedentes patológicos personales de hipertrofia prostática benigna en tratamiento farmacológico. Quien acude a valoración por tos de 2 meses de evolución no productiva, acompañado de pérdida de peso de aproximadamente $10 \mathrm{~kg}$ y alza térmica cuantificada de 39 grados centígrados, con esta sintomatología acude a hospital de segundo nivel donde es ingresado con diagnóstico de neumonía comunitaria pautándose tratamiento a base de ceftriaxona durante 3 días, completando de manera ambulatoria cefuroxima durante 7 días más. Al no haber respuesta al tratamiento clínico es derivado a la consulta externa de neumología de nuestro hospital.

A su llegada a nuestro servicio se realiza una tomografía de tórax simple (imagen 1)

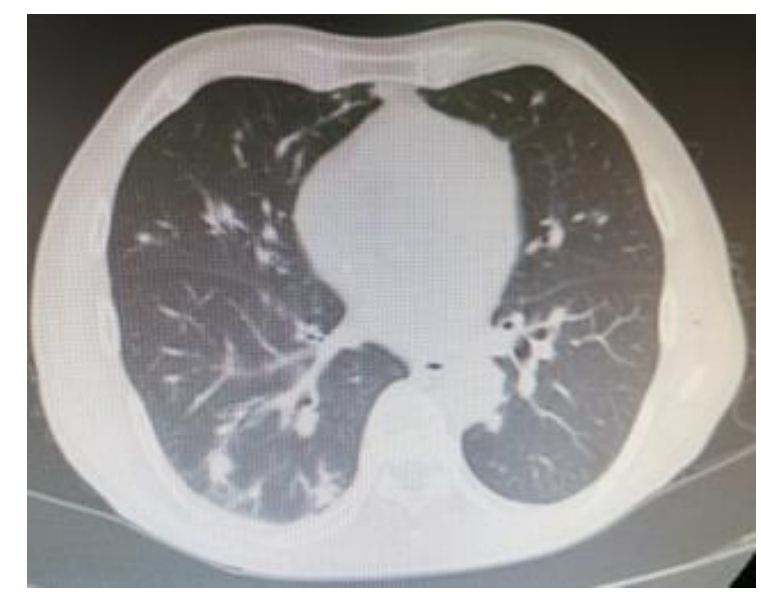

Imagen 1. TAC de tórax simple de ingreso, lesione difusas a nivel de campos pulmonares.

Observándose lesiones nodulares y micronodulares difusas a nivel de ambos campos pulmonares, con predominio derecho, por lo que se decide realizar broncoscopia diagnostica. En la broncoscopia se objetiva infiltración de toda la mucosa bronquial de predominio derecho desde carina traqueal, con citología, lavado y biopsia bronquial sospechoso de malignidad, con microbiología negativa. Debido a este hallazgo y que no se obtuvo un resultado definitivo por la broncoscopia, se decide realizar biopsia abierta para toma de muestras a descartar neoplasia pulmonar, tuberculosis pulmonar u otras causas. 
Durante su hospitalización paciente presenta hemoptisis por 3 días acompañado de alza térmica de 38.5 grados centígrados durante 2 días; posteriormente dolor precordial tipo opresivo de moderada intensidad con irradiación a hombro izquierdo, por lo que se solicita valoración al servicio de Cardiología quienes realizan exámenes y diagnostican Fibrilación Auricular, cardiovirtiendo con Amiodarona con respuesta favorable, y como hallazgo incidental foramen oval permeable.

Se realizó exámenes biometría hemática con leucocitos de 9.000, neutrófilos $73.1 \%$ hemoglobina: $12 \mathrm{~g} / \mathrm{dl}$ hematocrito: $36 \%$, glucosa: $187 \mathrm{mg} / \mathrm{dl}$ creatinina $0.99 \mathrm{mg} / \mathrm{dl}$, urea: 26 $\mathrm{mg} / \mathrm{dl}$, procalcitona: $0.11 \mathrm{ng} / \mathrm{dl}$. ANA, ANTIDNA C3-C4 perfil para SAF, ANTI CCP, ANTI RO- normales, ANCA C negativo, ANCA P negativo. ZIEHL en esputo negativo.

Se realizó biopsia abierta pulmonar a nivel de segmento 10 de pulmón derecho por minitoracotomia, con presencia de múltiples nodulaciones a nivel de porción basal del lóbulo inferior de pulmón derecho, de consistencia dura. Se palparon tumoraciones más pequeñas en todo el parénquima de lóbulo inferior. Siendo el resultado de la biopsia un diagnostico compatible con granulomatosis con poliangitis.

Inició tratamiento servicio de reumatología con ciclofosfamida durante 6 ciclos.

Tras cumplir sus 6 ciclos de inmunosupresor se realizó broncoscopia de control en la cual no se encontraron alteraciones.

El paciente evoluciona favorablemente desde el punto de vista clínico y tomográfico. (imagen 2).

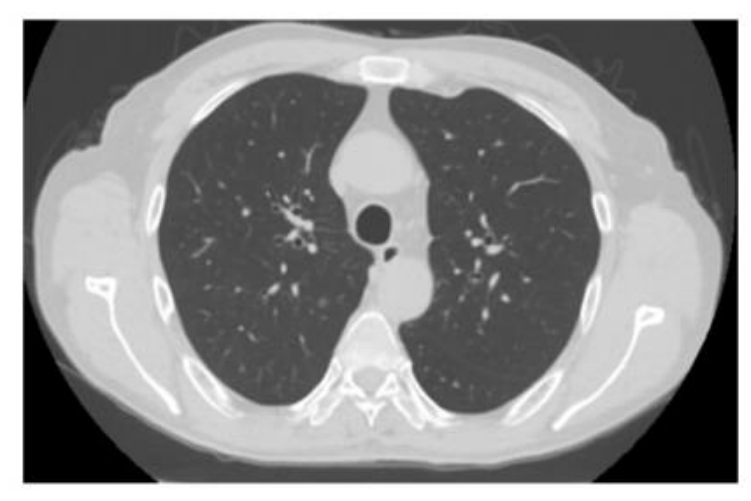

Imangen 2. TAC de torax simple de control: No hay evidencia de infiltrados ni consolidaciones

\section{DISCUSIÓN}

Las vasculitis son un conjunto heterogéneo de enfermedades sistémicas caracterizadas por la inflamación y en cierta medida destrucción de los vasos sanguíneos, por ende, afectan la perfusión o permeabilidad de dichos vasos, su etiología es desconocida. 
Dentro de la clasificación de las vasculitis, el consenso internacional de Chapel Hill del 2012, establece una agrupación de dichas enfermedades de la siguiente manera:

Vasculitis de grandes vasos, vasculitis de medianos vasos, vasculitis de pequeños vasos (asociadas a anticuerpos anti citoplasma de neutrófilos (ANCA), asociadas a complejos inmunes), vasculitis de vaso variable, vasculitis de un solo órgano, vasculitis asociadas a enfermedades sistémicas y vasculitis asociadas con una probable etiología. Aproximadamente el $10 \%$ de los pacientes con una GP típica clínicamente, tienen ANCA negativo. ${ }^{(6)}$

En el presente caso se trata de enfermedad inmunológica relacionada a pequeños vasos con anticuerpos anti citoplasma de neutrófilos negativos.

La enfermedad granulomatosa incluye afección a nivel ocular (pseudotumororbitario, escleritis nodular, dacriocistitis), auditivo (hipoacusia por infiltración granulomatosa de oído medio e interno), de vía aérea superior e inferior (destrucción nasal y de senos paranasales, estenosis subglótica, masas en parénquima pulmonar y bronquios) y del sistema nervioso central paquimeningitis o afección de la glándula hipófisis. ${ }^{(7)}$

La insuficiencia renal crónica se observa de 11 a $32 \%$ y es una manifestación de mal pronóstico, en especial cuando se presenta como glomerulonefritis rápidamente progresiva (8)

La afección pulmonar se da en el 50-90 \% de los pacientes, lo más destacado es una hemorragia alveolar que lleva a hemoptisis y ocurre en el 29-36 \% de los casos, varía en su gravedad, puede presentarse con una pequeña cantidad de sangre, hasta una hemorragia masiva con falla respiratoria aguda, en el 7.2-36\% de los pacientes en el momento del diagnóstico y se relaciona con un peor pronóstico. También se ven nódulos pulmonares, únicos o múltiples, que cambian de un lado a otro del parénquima pulmonar.

A nivel radiológico se encuentran infiltrados pulmonares nodulares, cavitaciones únicas o múltiples, opacidades en vidrio esmerilado bilaterales, un patrón reticulonodular, disminución del volumen pulmonar con alta incidencia de patrón en panal de abeja (73-84 $\%$ ). La mayoría de estas alteraciones se evidencian predominantemente en las regiones inferiores y externas del pulmón. ${ }^{(9)}$

Las características histopatológicas cardinales de GP incluyen una vasculitis necrotizante que afecta a las arteriolas, vénulas y capilares; inflamación granulomatosa; necrosis; microabscesos; y fibrosis, por ende, la fibrosis de las paredes vasculares puede resultar en estenosis u obliteración de los lúmenes. ${ }^{(10)}$

El diagnóstico definitivo de una granulomatosis con poliangitis se da al tomar una biopsia lesional y demostrar la presencia de una vasculitis granulomatosa necrosante (tejido pulmonar es más certero). El ANCA es un dato complementario y no debe sustituir el diagnóstico histológico de la biopsia ya que puede haber falsos positivos en infecciones y tumores. $^{(11)}$ 
En cuanto al pronóstico, los regímenes inmunosupresores han mejorado el pronóstico de modo que la supervivencia a 5 años es ahora del 70-80\%; Sin embargo, en solo el 75\% de los pacientes logran remisión. Las recaídas pueden surgir en aproximadamente el 50\% de los pacientes y la recurrencia dentro de los 18 meses a 15 años.

Los factores de riesgo para las recaídas incluyen la interrupción del tratamiento, la rápida disminución de los corticosteroides, y enfermedad recidivante previa ${ }^{(2)}$

El caso presentado en el presente artículo se trata de un paciente sin patología pulmonar crónica previa, en quien se decide realizar biopsia pulmonar abierta por cuadro de tos de 2 meses de evolución más pérdida de peso, en quien se sospechó Neoplasia; sin embargo, por resultado de biopsia se muestra imagen compatible con Vasculitis, de tal forma se reafirma lo que la literatura menciona que el diagnóstico definitivo se da mediante biopsia.

El tratamiento se divide en dos fases: de inducción a la remisión y de mantenimiento. En la primera es necesario el uso de terapia inmunosupresora intensiva: ciclofosfamida más esteroides para controlar la actividad de la enfermedad (tres a seis meses). En la fase de mantenimiento, menos intensa, se emplean inmunosupresores como azatioprina, metotrexate, micofenolato de mofetilo, entre otros; su objetivo es mantener la remisión y disminuir los efectos adversos asociados a la ciclofosfamida.

El tratamiento con ciclofosfamida y corticoides ha mejorado el pronóstico de las vasculitis, consiguiendo la remisión entre el 70-90\% de los casos. A pesar de ello, un 10\% de los pacientes no consigue alcanzar la remisión o presentan frecuentes recaídas y son considerados pacientes refractarios al tratamiento. Se han descrito múltiples tratamientos alternativos para los casos de Gp refractaria como: infliximab, etanercept, 15deoxyspergualina, leflunomida, sueros antilinfocitarios y rituximab; por desgracia no hay suficientes estudios que indiquen cuál es la mejor terapia alternativa. ${ }^{(12)}$

El patrón de referencia para GP severa es enfermedad renal con concentraciones de creatinina mayores de $5.6 \mathrm{mg} / \mathrm{dL}$ o insuficiencia inminente de otro órgano vital junto con síntomas constitucionales.

Para el tratamiento de la GP, posterior a un periodo promedio de tres a seis meses de tratamiento intensivo, la remisión se logra en 87 a $92.7 \%$ de los casos sin afección renal al momento del diagnóstico, siendo necesario iniciar manejo menos intensivo con la finalidad de evitar la recaída y los efectos adversos. ${ }^{(13)}$

El tratamiento en el caso presentado se objetivo en terapia inmunospresora de seis ciclos de ciclofosfamida con buena evolución clínica.

\section{CONCLUSIONES}

La GP es un trastorno multisistémico, que puede relacionarse con una importante morbilidad y mortalidad. Su etiología sigue siendo desconocida, pero su característica principal está dada por la inflamación particularmente de la vía aérea superior, inferior y a nivel renal. Aunque la GP se ha asociado a la presencia de ANCA positivos, no siempre es indicativo de 
enfermedad, como es el caso expuesto en este artículo. Por ello se hace indispensable la realización de examen histológico del tejido lesional para confirmar el diagnóstico. Si no se la trata casi siempre causa la muerte dentro del primer año de haber sido diagnosticada. El pronóstico es reservado y depende de la manifestación y tiempo de diagnóstico. La enfermedad renal es rápidamente progresiva y se asocia con hemorragia pulmonar, que es la de peor pronóstico a corto plazo.

\section{Referencias bibliográficas.}

CHENG-WEI LU, X. F.-D.-M.-N.-L. A case repor of orbit, ocular association and the lung in granulomatosis with polyangiitis: A diagnostic challenge, Experimental and therapeutic medicine, 2017, 3338-3340.

Alfonso García-Luna, Víctor Hugo Nez-Esquivel, Francisco López-Baca, Jorge MoraConstantino. Granulomatosis con poliangitis. 2015, 47-51.

VASCULITIS ANCA P POSITIVO.pdf - Repositorio Universidad Técnica de Ambato. 2016 enero. $2019 \quad$ marzo 20. http://repositorio.uta.edu.ec/bitstream/123456789/18014/2/VASCULITIS\%20ANCA\% 20P\%20POSITIVO.pdf

León-Ortiz AG, Guamán-Crespo JO, Sánchez-Zúñiga MJ, Carrillo-Esper R. Granulomatosis con poliangitis, granulomatosis de Wegener. Med Int Méx. 2017 mayo;33(3):421-426.

Luis Felipe FLORES-Suárez. Marco A. Alba. Heidegger Mateos-Toledo. Natllely Ruiz. Pulmonary Involvement in Systemic Vasculitis. Curr Rheumatol Rep, 2017, 2-8

Diana Carolina Jiménez Camacho. Granulomatosis con Poliangitis (Wegener). REVISTA MEDICA DE COSTA RICA Y CENTROAMERICA LXXII. 2015, (615) 233 - 237.

Luisa Paz Sendín, Lorenzo Gómez Morales, Gertrudis Hernández González, Raúl González Torres, Arturo Munster Infante y Luis Rodríguez Rivera. GRANULOMATOSIS DE WEGENER CON MANIFESTACIONES NEUROLÓGICAS. A PROPÓSITO DE 1 CASO. Rev cubana Med 2015; 40(3):223-7

Olga Vera-Lastra, Arturo Olvera-Acevedo, Alejando McDonald-Vera, Manuel PachecoRuelas y José Arturo Gayosso-Rivera. Granulomatosis de Wegener, abordaje diagnóstico y terapéutico. 2015, 121-127.

Simón Hoyos Patiño. Granulomatosis con Poliangeítis: actualización y conceptos claves. Revista de Cuba de Reumatología. 2016,36-41. 
Joseph P. Lynch III, Ariis Derhovanessian, Henry Tazelaar, John A. Belperio. Granulomatosis with Polyangiitis (Wegener's Granulomatosis): Evolving Concepts in Treatment. Semin Respir Crit Care Med 2018; 39:434-458.

Masanori Harada, Shiro Imokawa, Satoru Miwa, Fumiya Nihashi, Yuya Aono, Yusuke Amano, Tomohiro Uto, Jun Sato and Takafumi Suda. Chronic pulmonary aspergillosis may cause eosinophilic granulomatosis with polyangiitis via allergic bronchopulmonary aspergillosis. Oxford Medical Case Reports, 2019;2, 63-66.

Vera-Lastra O, Olvera-Acevedo A, McDonald-Vera A, Pacheco-Ruelas M, Gayosso-Rivera JA. Granulomatosis de Wegener, abordaje diagnóstico y terapéutico. 2014, 145(2)

Olga Vera-Lastra, Arturo Olvera-Acevedo, Alejando McDonald-Vera, Manuel PachecoRuelasc y José Arturo Gayosso-Rivera. Granulomatosis de Wegener, abordaje diagnóstico y terapéutico. 2015, 121-127. 


\section{PARA CITAR EL ARTÍCULO INDEXADO.}

Valdiviezo Allauca, N., Chávez Berrones, M., \& Merino Guerrero, C. (2019). Granulomatosis con Poliangitis: presentación de un caso. Ciencia Digital, 3(3.3), 286-294. https://doi.org/10.33262/cienciadigital.v3i3.3.821

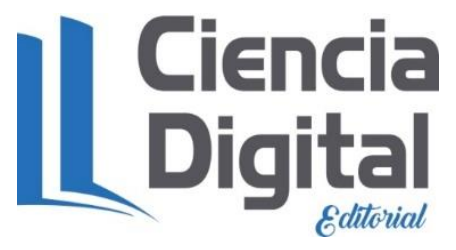

El artículo que se publica es de exclusiva responsabilidad de los autores y no necesariamente reflejan el pensamiento de la Revista Ciencia Digital.

El artículo queda en propiedad de la revista y, por tanto, su publicación parcial y/o total en otro medio tiene que ser autorizado por el director de la Revista Ciencia Digital.
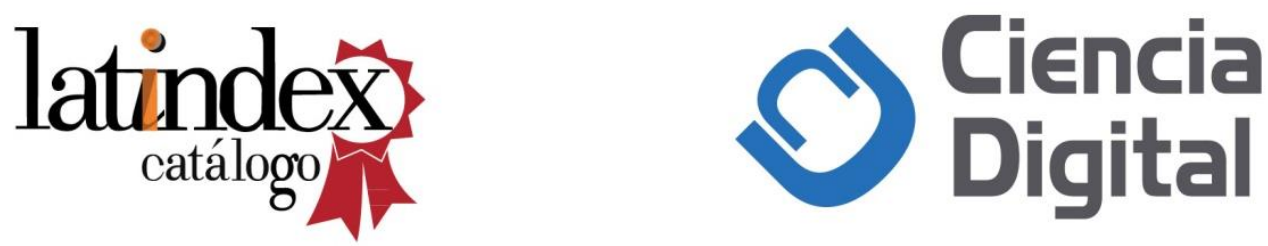